\title{
The benefits of hypothermic machine preservation and short cold ischemia times in deceased donor kidneys
}

Kox JJHFM, MD ${ }^{1}$, Moers C, MD, $\mathrm{PhD}^{2}$, Monbaliu D, MD, $\mathrm{PhD}^{3}$, Strelniece A, MSc ${ }^{4}$, Treckmann J, MD 5 , Jochmans I, MD, $\mathrm{PhD}^{3}$, Leuvenink HGD, $\mathrm{PhD}^{2}$, Van Heurn LWE, MD, $\mathrm{PhD}^{1}$, Pirenne J, MD, $\mathrm{PhD}^{3}$, Paul A, MD, $\mathrm{PhD}^{5}$, Ploeg RJ, MD, PhD 2,6

${ }^{1}$ Department of Surgery, Maastricht University Medical Center, Maastricht, Netherlands

${ }^{2}$ Department of Surgery - Organ Donation and Transplantation, University Medical Center Groningen, University of Groningen, Groningen, Netherlands

${ }^{3}$ Abdominal Transplantation, Department of Microbiology and Immunology, KU Leuven, Leuven; Abdominal Transplant Surgery, University Hospitals Leuven, Leuven, Belgium

${ }^{4}$ Eurotransplant International Foundation, Leiden, Netherlands

${ }^{5}$ Department of General, Visceral and Transplantation Surgery, University Hospital Essen, Essen, Germany

${ }^{6}$ Oxford Transplant Centre, Oxford, United Kingdom 
Correspondence Information

Address: $\quad$ Maastricht University Medical Center

Jasper Kox, 2.G1.027

P.O. Box 5800, 6202 AZ Maastricht

The Netherlands

E-mail: $\quad$ j.kox@ maastrichtuniversity.nl

Clinical trial notation

ISRCTN83876362

\section{Authorship}

Authorship statement

Kox JJHFM: Writing of the paper, participated in data analysis

Moers C: Writing of the paper, participated in data analysis, participated in research design, participated in performance of the research

Monbaliu D: Participated in research design, participated in performance of the research, participated in paper revision

Strelniece A: Participated in data analysis, participated in paper revision

Treckmann J: Participated in research design, participated in performance of the research, participated in paper revision

Jochmans I: Participated in research design, participated in performance of the research, participated in paper revision

Leuvenink HGD: Participated in research design, participated in performance of the research, participated in paper revision 
Van Heurn LWE: Participated in research design, participated in performance of the research, participated in paper revision

Pirenne J: Participated in research design, participated in performance of the research, participated in paper revision

Paul A: Participated in research design, participated in performance of the research, participated in paper revision

Ploeg RJ: Participated in research design, participated in performance of the research, participated in paper revision

\section{Disclosures}

The authors declare that they have no conflicts of interest relevant for the present study.

\section{Funding}

The clinical study on which the current analyses are based was financially supported by Organ Recovery Systems, Itasca, IL, USA. 


\section{Abbreviations}

CIT, cold ischemia time

CS, cold storage

DBD, donation after brain death

DCD, donation after circulatory death

DGF, delayed graft function

ECD, expanded criteria donor

HLA, human leukocyte antigen

HMP, hypothermic machine preservation

HR, hazard ratio

HSP, heat-shock protein

HTK, histidine-tryptophan-ketoglutarate

KLF2, Kruppel-like factor type 2

OR, odds ratio

SCD, standard criteria donor

SD, standard deviation

SRTR, Scientific Registration of Transplant Recipients

UW, University of Wisconsin 


\begin{abstract}
BACKGROUND

Hypothermic machine perfusion (HMP) of deceased donor kidneys is associated with better outcome when compared to static cold storage (CS). Nevertheless, there is little evidence whether kidneys with short cold ischemia time (CIT) also benefit from HMP and whether HMP can safely extend CIT.
\end{abstract}

\title{
METHODS
}

We analysed prospectively collected data from the Machine Preservation Trial, an international randomized controlled trial. 752 Consecutive renal transplants were included: 1 kidney of each of the 376 donors was preserved by HMP, the contralateral organ was preserved by CS.

\section{RESULTS}

The mean CIT was 15:05 hours (SD 4:58 hours). A subgroup analysis was performed, groups were based on CIT duration: 0-10 hours, 10-15 hours, 15-20 hours, or 20 hours and more. Delayed graft function (DGF) incidence in the subgroup with up to 10 hours CIT was $6.0 \%$ $(\mathrm{N}=3 / 50)$ in the HMP arm and 28.1\% $(\mathrm{N}=18 / 64)$ in the CS arm (univariable $\mathrm{p}=0.002$, multivariable OR 0.02, $\mathrm{p}=0.007$ ). CIT remained an independent risk factor for DGF for machine perfused kidneys recovered from DBD donors OR 1.06, 95\% CI 1.017-1.117, $\mathrm{p}=0.008)$, DCD donors (OR 1.13, 95\% CI 1.035-1.233, p=0.006) and ECD donors (OR 1.14, 95\% CI 1.057-1.236, $\mathrm{p}=0.001)$. 


\section{CONCLUSION}

In conclusion, HMP resulted in remarkably lower rates of DGF in renal grafts that were transplanted after a short CIT. Also, CIT remained an independent risk factor for DGF in HMP-preserved kidneys. 


\section{Introduction}

Kidney transplantation is the preferred treatment for end-stage renal disease. Transplantation numbers are limited by a shortage of organ donors. To utilise all available deceased donor organs, optimal preservation of allografts between procurement and transplantation is paramount. In kidney transplantation, preservation of deceased donor kidneys by hypothermic machine perfusion (HMP) has been associated with better posttransplant outcome than static cold storage (CS). ${ }^{1-6}$ Other benefits of HMP include the opportunity to study renovascular and perfusate parameters. $^{7-10}$

Hypothermically preserved grafts still sustain cold ischemic injury. During cold ischemia, there is a build-up of toxic substances, lysosomal instability and cellular oedema, through inactivation of $\mathrm{Na}+\mathrm{K}+$ ATPase pumps, resulting in cold ischemic damage. ${ }^{11,12}$ Longer CIT in renal transplantation is associated with the occurrence of DGF, PNF and decreased graft survival. ${ }^{13,14}$ Especially marginal kidneys, kidneys from ECD and DCD donors, have shown to be more sensitive to cold ischemia. ${ }^{13,15}$ These processes are responsible for an increased risk of delayed graft function of the transplanted kidney and have a negative effect on the long-term graft and patient survival, particularly if the cold ischemia time is prolonged. ${ }^{16}$

It is generally presumed that HMP only benefits marginal kidneys or kidneys that sustain significant ischemic injury and that kidneys that are transplanted after a short cold ischemic period do not benefit from this preservation technique., ${ }^{2,17}$ Another general clinical presumption is that cold ischemia time only has a small effect on outcome when a kidney is preserved by HMP, ie that a kidney is "safer" on the machine than it is in cold storage. The aim of this study was to test these 2 hypotheses. 


\section{Materials and Methods}

\section{MACHINE PRESERVATION TRIAL}

We performed a post hoc data analysis of the Machine Preservation Trial data. ${ }^{4,5}$ This international multi-center randomized controlled trial was conducted in the Netherlands, Belgium and in the North Rhine-Westphalia federal state of Germany and investigated the effects of machine perfusion on delayed graft function (DGF), graft survival and other relevant end points. In the study, all consecutive donation after circulatory death (DCD) category III or IV and donation after brain death (DBD) kidney pairs were randomized in such a way that 1 kidney of each pair underwent HMP and the contralateral organ was cold stored.

\section{STUDY POPULATION AND INCLUSION CRITERIA}

376 DBD or DCD kidney donors aged 16 years or older were included. DCD donors were in Maastricht category III (awaiting circulatory death after withdrawal of treatment). ${ }^{18}$ Kidney pairs were only included if both organs were transplanted into 2 different recipients. Exclusion criteria were multi-organ transplantation in 1 or both recipients and death of a recipient within 1 week after transplantation (follow-up of 7 days was required for the primary end point: DGF).

\section{PRESERVATION}

All included kidneys underwent in situ vascular flush with either cold storage University of Wisconsin (CS-UW) or histidine-tryptophan-ketoglutarate (HTK) solution, based on local retrieval team practices. Kidney pairs were flushed out with the same solution. The HMPassigned kidneys were connected to the perfusion machine (LifePort Kidney Transporter, Organ Recovery Systems, Itasca, IL, USA) for the whole duration of the preservation period. 
HMP was done with machine preservation solution (Kidney Preservation Solution-1, KPS-1, Organ Recovery Systems) at 1-8 degrees Celsius and a set perfusion pressure of 30mmHg.

\section{STATISTICAL ANALYSIS}

The patient cohort was divided into 4 different groups, based on the duration of the cold ischemia time. The empirically chosen groups are 0-10 hours, 10-15 hours, 15-20 hours or greater than 20 hours.

Continuous variables are presented as mean \pm standard deviation (SD) if there is a normal distribution and as median and range otherwise.

Differences in baseline characteristics between donor types were tested with independentsamples t-tests for normally distributed continuous variables, Mann-Whitney U-tests for nonnormally distributed continuous variables and $\chi^{2}$-tests for categorical variables.

DGF was defined as any dialysis requirement in the first week after transplantation. Deathcensored graft survival was calculated with the Kaplan-Meier survival analysis method. Multivariable survival analysis was done with the Cox proportional hazards analysis, using the same covariates as in previous analyses with these data. The choice of these covariates was based on relevant literature. The risk of DGF was modelled with multivariable logistic regression analysis. ${ }^{1,4-6,19}$ Variables used in the Cox proportional hazards analysis and multivariable logistic regression analysis were: HMP or CS, donor and recipient age, time spent on the waiting list, panel-reactive antibody percentage, cold ischemia time, expanded or standard criteria donation, total number of HLA mismatches and the number of previous transplants. To further explore the impact of the preservation method on the effect that the 
CIT had on the risk of DGF, an interaction term (preservation method x CIT) was added to the multivariable logistic regression model for DGF.

All analyses were performed with SPSS (Statistical Package for the Social Sciences) version 22.0. A $p$ value of $\leq 0.05$ was considered to indicate statistical significance.

\section{EXPANDED CRITERIA DONATION}

Expanded criteria donation was defined as a donor age over 60 years or a donor age between 50 and 60 years with at least 2 of the following: a cerebrovascular cause of death, a serum creatinine level greater than $132 \mu \mathrm{mol} / \mathrm{L}(1.5 \mathrm{mg} / \mathrm{dL})$, or a history of hypertension. ${ }^{5,20}$

\section{ETHICAL APPROVAL}

Approval for this international trial was obtained from ethics review boards in each trial region and from the Eurotransplant Ethical Advisory Committee and the Eurotransplant Kidney Advisory Committee.

\section{Results}

Between November 1, 2005 and September 12, 2007, a total of 376 consecutive kidney donors were included and a total of 752 kidneys were randomly assigned to either HMP or CS.

As a result of the study's strictly paired design, donor characteristics were exactly the same for both treatment arms. Median donor age was 49.6 years (range 16-81). There were 294 DBD donors and 82 DCD category III donors. 105 Were expanded criteria donors, the other 271 were standard criteria donors. 
Table 1 shows the previously reported overall baseline characteristics. ${ }^{5}$ For the HMP group, median recipient age was 53 years (range 11-79) and for the CS group, this was 52 (range 279). Cold ischemia times were 15 hours and 10 minutes (range 3-47 hours) and 15 hours and 5 minutes (range 3-30 hours), respectively. Table 2 shows baseline characteristics for each CIT subgroup.

\section{THE EFFECT OF HMP ON DGF ACROSS DIFFERENT COLD ISCHEMIA TIMES}

The effect of HMP on the incidence of DGF is shown in Table 3. We have performed a subgroup analysis after empirically dividing the dataset into 4 CIT groups (less than 10 hours, 10-15 hours, 15-20 hours and more than 20 hours). The impact of HMP in this dataset was largest in the subgroup that had sustained the least amount of cold ischemic injury, a CIT less than 10 hours. In this subgroup, the incidence of DGF after transplantation was $6.0 \%$ (3 out of 50) for kidneys preserved by HMP, whereas after CS this incidence was $28.1 \%$ (18 out of 64). This difference was statistically significant in both a univariable analysis $(p=0.002)$ and a multivariable analysis (odds ratio $(\mathrm{OR})=0.015, \mathrm{p}=0.007)$. In the group with the longest cold ischemic period, there was also a difference in DGF occurrence between the 2 preservation methods. Although this difference did not reach statistical significance, there was a definite trend, especially in the multivariable analysis. In this subgroup with the longest CIT, the OR for the risk of developing DGF was 0.335 (95\% CI 0.11-1.015), $\mathrm{p}=0.053$. In the other subgroups, that sustained 10-15 and 15-20 hours of cold ischemia, the differences in DGF incidence between CS and HMP preserved grafts were small. Full data are provided in Table S1 ( $<10$ hrs), Table S2 (10-15 hrs), Table S3 (15-20 hrs) and Table S4 (>20 hrs) (SDC, http://links.lww.com/TP/B552). 
THE EFFECT OF HMP ON 3 YEAR GRAFT SURVIVAL

Kaplan-Meier and multivariable Cox proportional hazards analysis was performed to investigate whether the observed differences in DGF incidence would lead to a long-term effect of HMP versus CS on graft survival in each of the 4 CIT groups. For HMP and CS respectively, the graft survival rates were $88.0 \%$ vs $81.3 \%$ for $0-10$ hours of CIT, $91.9 \%$ vs $90.1 \%$ for $10-15$ hours, $93.8 \%$ vs $88.5 \%$ for $15-20$ hours and $84.9 \%$ vs $83.7 \%$ for more than 20 hours of cold ischemia. There was no statistically significant 3 year graft survival benefit for HMP preserved kidneys in any of the 4 subgroups. Kaplan-Meier graphs are shown in Figure 1.

\section{THE EFFECT OF CIT ON THE RISK OF DGF DURING HMP VERSUS CS}

In a multivariable logistic regression analysis modelling the risk of DGF, the OR of each additional hour of CIT during CS overall was 1.08 (95\% confidence interval (CI) 1.03-1.14, $\mathrm{p}=0.004)$. The multivariable OR of each hour of CIT during HMP overall was also 1.08 (95\% CI 1.04-1.14, $\mathrm{p}=0.003)$. Multivariable interaction analysis showed that HMP vs CS did not change the effect that the CIT had on the risk of DGF (nonsignificant interaction with no evidence of effect modification, $\mathrm{p}=0.797$ ). Next, multivariable analyses were performed for various donor type subgroups: The multivariable OR of an additional hour of CIT during HMP was 1.06 (95\% CI 1.017- 1.117, p=0.008) for DBD kidneys and 1.13 (95\% CI 1.0351.233, $\mathrm{p}=0.006)$ for DCD grafts. For ECD organs, the OR was 1.14 (95\% CI 1.057-1.236, $\mathrm{p}=0.001)$

\section{THE EFFECT OF PERFUSION SOLUTION}

Kidney pairs were flushed out with either CS-UW $(n=226)$, HTK $(n=143)$ or another perfusion solution $(n=7)$. To rule out effects of the preservation solution in our analyses, we 
compared groups for the prevalence of HTK and CS-UW use and performed both univariate and multivariate analyses.

For 0-10 hours of CIT, 55\% of the kidney pairs were flushed with CS-UW. For 10-15, 15-20 and $>20 \mathrm{hrs}$, the percentage of kidney pairs flushed with CS-UW was $62 \%, 59 \%$ and $55 \%$, respectively. There was no significant difference in the number of CS-UW flushed kidney pairs throughout the 4 CIT groups $\left(\chi^{2}, \mathrm{p}=0.48\right)$.

In cold storage preserved kidneys, there was no significant effect of the preservation solution for either DBD ( $\mathrm{p}=0.760)$ or DCD kidneys $(\mathrm{p}=0.829)$. The multivariate OR for DGF when comparing CS-UW to HTK as a preservation solution during cold storage was $1.481(0.797$ 2.752, $\mathrm{p}=0.214)$.

\section{Discussion}

Optimal preservation of deceased donor organs is paramount and sustains organ viability in an ex vivo setting. ${ }^{21}$ Hypothermic machine perfusion has been associated with lower rates of delayed graft function and better long term graft survival than its more widely employed counterpart, static cold storage. ${ }^{4,5,22-24}$

Interestingly, in current clinical practice, there are some general assumptions about the benefits and limitations of hypothermic machine perfusion, based on the evidence that supports HMP as a more beneficial preservation method. ${ }^{1-6,19,25}$ This may have a paradoxical effect: the underestimation of some of the most well-known risk factors for delayed graft function, especially cold ischemia time, during HMP. 
Our data suggest that these general assumptions may be a misconception about the protective effect of HMP. It is thought by many that the duration of cold ischemia time can be relatively safely extended if kidneys are machine perfused. We have shown that, quite surprisingly, HMP actually yields the largest benefit in kidneys which are preserved for a relatively short period, less than 10 hours of CIT. There was also a trend towards a relevant beneficial effect of HMP in kidneys that are stored for more than 20 hours. However, in this subgroup the differences in DGF incidence between HMP and CS were not significant, which is likely due to the relatively small number of organs in the prolonged CIT subgroup. In the other 2 subgroups, the relative effect of HMP vs CS on the risk of DGF was less pronounced and, with limited numbers in each group, not statistically significant. Irrespective of a potential benefit of HMP, the present study shows that in HMP preserved kidneys it is also essential to limit CIT as much as possible. Previous studies have shown that CIT is an independent risk factor for DGF during CS. ${ }^{13,16,26}$ In a multivariable data analysis, we have shown that the detrimental effect of CIT during HMP has a similar magnitude as during CS: Regardless of whether the kidney is on the pump or in a bag, every additional hour of cold ischemia increases the odds for developing delayed graft function with $8 \%$.

In current clinical practice, HMP is used most often to preserve kidneys that are expected to undergo a long period of cold ischemia. The resulting lack of data on the beneficial effect of HMP versus CS for short preservation times may have led to the common presumption that there is no additional value of HMP in kidneys that are transplanted quickly. Gill et al have reported data from the Scientific Registration of Transplant Recipients (SRTR) ${ }^{27}$ which showed a significant beneficial effect of HMP in kidneys recovered from SCD, ECD and DCD donors, with various CIT. In contrast with our findings, data from these 94,709 transplant recipients, showed no significant benefit in a subgroup of kidneys from ECD or 
DCD donors, transplanted after a short CIT. There are several differences in study design which may explain this discrepancy. First, ours was a prospectively randomized study, with no selection of which kidneys were pumped and which were cold stored. Second, in our study kidneys were all machine perfused during the entire preservation period, whereas most renal grafts in the SRTR region were only on HMP for a few hours just prior to transplantation, preceded by a longer period of CS. Third, our dataset was not large enough to also create meaningful subgroups of ECD and DCD kidneys out of the subgroup of kidneys with a short CIT.

The present analysis of prospectively collected data shows that there is a clear cut and quite remarkable benefit of HMP for kidneys that undergo no more than 10 hours of cold ischemia. In this subgroup, the incidence of DGF in kidneys preserved by HMP was much lower than for cold stored organs. Such a relatively large benefit of HMP over CS in terms of DGF reduction has not been reported before. The underlying mechanisms are unknown and different causes can only be hypothesized. The beneficial effect could perhaps be attributed to vasodilatation and a lower vascular resistance after HMP. De Vries et al have shown that the renal resistance of a recently procured and cooled kidney usually decreases in the first 30-60 minutes of machine perfusion; after this period renal resistance hardly decreases any further. ${ }^{8}$ Vasodilatation may lead to improved reperfusion after transplantation with potentially beneficial effects on renal cortical perfusion, oxygen and nutrient delivery after transplantation. When CIT is long, renal oedema inhibits capillary flow in both HMP and CS and the benefits of the initial vasodilatation may be cancelled out.

In addition, HMP may in theory help to better wash out vasoactive and inflammatory molecules and limit inflammatory reactions inside the graft after transplantation. ${ }^{28,29} \mathrm{~A}$ 
relatively short period of HMP is likely to provide an already quite extensive wash out, with longer HMP times perhaps not eliminating much more of these components. During HMP, there is a more continuous exchange of electrolytes and other molecules between the preservation solution and the intracellular space, compared to static CS. Because of this, accumulation of pro-inflammatory cytokines such as interleukin-1, interleukin-6, tumour necrosis factor- $\alpha$ and tumour growth factor- $\beta$, heat-shock proteins (mostly HSP-60 and HSP70) and complement factor 3 a may be partially prevented. ${ }^{7,28,30-32}$ Inhibition of the innate immune system through toll-like receptors and the complement system could be 1 of the underlying mechanisms of action of HMP..$^{28,31}$

In addition, Kruppel-like factor 2 may play a pivotal role in protection of the vascular endothelium during HMP. ${ }^{33}$ KLF2 is a transcription factor that plays a role in the development and activation of the vascular endothelium. It also attenuates induction of proinflammatory molecules like E-selectin and vascular cell adhesion molecule 1 (VCAM-1), regulates gene expression of thrombomodulin and activates of endothelial nitric oxide synthase (eNOS) potently. ${ }^{34,35}$ KLF2 is upregulated by pulsatile endothelial shear stress. ${ }^{35,36}$ This may explain part of the protective effect of HMP and this particular mechanism of benefit is not likely to be stronger after longer periods of machine perfusion. The provision of pulsatile flow upregulates KLF2 to protect the vascular endothelium of an organ during the preservation period. ${ }^{33}$

HMP may also have potential deleterious effects on the preserved graft. Shear stress can cause vascular injury, particularly endothelial injury, as a result of pressure driven flow of cold perfusate. Oedema may occur as a result of ischemic injury, resulting in higher vascular resistance. ${ }^{37,38}$ This may further intensify perfusion injury. These deleterious effects seem to 
be most pronounced during prolonged perfusion and in kidneys that are most vulnerable to cold ischemia time. Gill et al have shown that in DCD donors, HMP and CS have comparable results after 36 hours of $\mathrm{CIT}^{27}$ This may be in part be explained by the hypothesis that beneficial effects of HMP diminish over time and eventually even are outweighed by the method's deleterious effects.

In conclusion, the overall superiority of HMP over CS as a preservation method for renal grafts is evident and has been shown before by many studies. In the present analysis of data obtained in a large randomized controlled trial, we have shown that HMP is beneficial when cold ischemia times are short. Also, we have found that cold ischemia time remains an independent and equally relevant risk factor for DGF when kidneys are preserved by HMP instead of CS.

\section{LIMITATIONS}

We have performed a post hoc data analysis of a randomized controlled trial. Hence, common limitations of post hoc analyses also apply to the present study. One of the most important issues is that due to the division of original data into 4 subgroups, statistical power diminishes. As a result of relatively small numbers per subgroup, we may for example not have picked up differences in graft survival between HMP and CS preserved kidneys, which were evident in the overall study group that had a larger sample size. 


\section{References}

1. Gallinat A, Moers C, Smits JM, et al. Machine perfusion versus static cold storage in expanded criteria donor kidney transplantation: 3-year follow-up data. Transpl Int. 2013;26(6):E52-53.

2. Gallinat A, Moers C, Treckmann J, et al. Machine perfusion versus cold storage for the preservation of kidneys from donors $>/=65$ years allocated in the Eurotransplant Senior Programme. Nephrol Dial Transplant. 2012;27(12):4458-4463.

3. Jochmans I, O'Callaghan JM, Pirenne J, Ploeg RJ. Hypothermic machine perfusion of kidneys retrieved from standard and high-risk donors. Transpl Int. 2015;28(6):665676.

4. Moers C, Pirenne J, Paul A, Ploeg RJ, Machine Preservation Trial Study G. Machine perfusion or cold storage in deceased-donor kidney transplantation. $N$ Engl J Med. 2012;366(8):770-771.

5. Moers C, Smits JM, Maathuis MH, et al. Machine perfusion or cold storage in deceased-donor kidney transplantation. N Engl J Med. 2009;360(1):7-19.

6. Treckmann J, Moers C, Smits JM, et al. Machine perfusion versus cold storage for preservation of kidneys from expanded criteria donors after brain death. Transpl Int. 2011;24(6):548-554.

7. De Deken J, Kocabayoglu P, Moers C. Hypothermic machine perfusion in kidney transplantation. Curr Opin Organ Transplant. 2016;21(3):294-300.

8. de Vries EE, van Smaalen TC, Boer J, et al. Measurement of renovascular circulating volume during hypothermic organ perfusion. Transplantation. 2013;95(9):1100-1104.

9. van Smaalen TC, Hoogland ER, van Heurn LW. Machine perfusion viability testing. Curr Opin Organ Transplant. 2013;18(2):168-173. 
10. Hoogland ER, de Vries EE, Christiaans MH, Winkens B, Snoeijs MG, van Heurn LW. The value of machine perfusion biomarker concentration in DCD kidney transplantations. Transplantation. 2013;95(4):603-610.

11. Salahudeen AK. Cold ischemic injury of transplanted kidneys: new insights from experimental studies. Am J Physiol Renal Physiol. 2004;287(2):F181-187.

12. Weinberg JM. The cell biology of ischemic renal injury. Kidney Int. 1991;39(3):476500.

13. van der Vliet JA, Warle MC, Cheung CL, Teerenstra S, Hoitsma AJ. Influence of prolonged cold ischemia in renal transplantation. Clin Transplant. 2011;25(6):E612616.

14. Dittrich S, Groneberg DA, von Loeper J, et al. Influence of cold storage on renal ischemia reperfusion injury after non-heart-beating donor explantation. Nephron Exp Nephrol. 2004;96(3):e97-102.

15. Summers DM, Johnson RJ, Hudson A, Collett D, Watson CJ, Bradley JA. Effect of donor age and cold storage time on outcome in recipients of kidneys donated after circulatory death in the UK: a cohort study. Lancet. 2013;381(9868):727-734.

16. van der Vliet JA, Warle MC. The need to reduce cold ischemia time in kidney transplantation. Curr Opin Organ Transplant. 2013;18(2):174-178.

17. Brat A, Pol RA, Leuvenink HG. Novel preservation methods to increase the quality of older kidneys. Curr Opin Organ Transplant. 2015;20(4):438-443.

18. Kootstra G, Daemen JH, Oomen AP. Categories of non-heart-beating donors. Transplant Proc. 1995;27(5):2893-2894.

19. Jochmans I, Moers C, Smits JM, et al. Machine perfusion versus cold storage for the preservation of kidneys donated after cardiac death: a multicenter, randomized, controlled trial. Ann Surg. 2010;252(5):756-764. 
20. Saidi RF, Elias N, Kawai T, et al. Outcome of kidney transplantation using expanded criteria donors and donation after cardiac death kidneys: realities and costs. Am J Transplant. 2007;7(12):2769-2774.

21. Maathuis MH, Leuvenink HG, Ploeg RJ. Perspectives in organ preservation. Transplantation. 2007;83(10):1289-1298.

22. Kaths JM, Paul A, Robinson LA, Selzner M. Ex vivo machine perfusion for renal graft preservation. Transplant Rev (Orlando). 2017.

23. Wight J, Chilcott J, Holmes M, Brewer N. The clinical and cost-effectiveness of pulsatile machine perfusion versus cold storage of kidneys for transplantation retrieved from heart-beating and non-heart-beating donors. Health Technol Assess. 2003;7(25):1-94.

24. Wight JP, Chilcott JB, Holmes MW, Brewer N. Pulsatile machine perfusion vs. cold storage of kidneys for transplantation: a rapid and systematic review. Clin Transplant. 2003;17(4):293-307.

25. van der Vliet JA, Kievit JK, Hene RJ, Hilbrands LB, Kootstra G. Preservation of nonheart-beating donor kidneys: a clinical prospective randomised case-control study of machine perfusion versus cold storage. Transplant Proc. 2001;33(1-2):847.

26. Debout A, Foucher Y, Trebern-Launay K, et al. Each additional hour of cold ischemia time significantly increases the risk of graft failure and mortality following renal transplantation. Kidney Int. 2015;87(2):343-349.

27. Gill J, Dong J, Eng M, Landsberg D, Gill JS. Pulsatile perfusion reduces the risk of delayed graft function in deceased donor kidney transplants, irrespective of donor type and cold ischemic time. Transplantation. 2014;97(6):668-674.

28. Tozzi M, Franchin M, Soldini G, et al. Impact of static cold storage VS hypothermic machine preservation on ischemic kidney graft: inflammatory cytokines and adhesion 
molecules as markers of ischemia/reperfusion tissue damage. Our preliminary results. Int J Surg. 2013;11 Suppl 1:S110-114.

29. Hosgood SA, Bagul A, Nicholson ML. Minimising cold ischaemic injury in an experimental model of kidney transplantation. Eur J Clin Invest. 2011;41(3):233-240.

30. Zhang Y, Fu Z, Zhong Z, et al. Hypothermic Machine Perfusion Decreases Renal Cell Apoptosis During Ischemia/Reperfusion Injury via the Ezrin/AKT Pathway. Artif Organs. 2016;40(2):129-135.

31. Liu Z, Zhong Z, Lan J, et al. Mechanisms of Hypothermic Machine Perfusion to Decrease Donation After Cardiac Death Graft Inflammation: Through the Pathway of Upregulating Expression of KLF2 and Inhibiting TGF-beta Signaling. Artif Organs. 2016.

32. Damman J, Bloks VW, Daha MR, et al. Hypoxia and Complement-and-Coagulation Pathways in the Deceased Organ Donor as the Major Target for Intervention to Improve Renal Allograft Outcome. Transplantation. 2015;99(6):1293-1300.

33. Gracia-Sancho J, Villarreal G, Jr., Zhang Y, et al. Flow cessation triggers endothelial dysfunction during organ cold storage conditions: strategies for pharmacologic intervention. Transplantation. 2010;90(2):142-149.

34. SenBanerjee S, Lin Z, Atkins GB, et al. KLF2 Is a novel transcriptional regulator of endothelial proinflammatory activation. J Exp Med. 2004;199(10):1305-1315.

35. Lin Z, Kumar A, SenBanerjee S, et al. Kruppel-like factor 2 (KLF2) regulates endothelial thrombotic function. Circ Res. 2005;96(5):e48-57.

36. Parmar KM, Larman HB, Dai G, et al. Integration of flow-dependent endothelial phenotypes by Kruppel-like factor 2. J Clin Invest. 2006;116(1):49-58. 
37. Hosgood SA, Mohamed IH, Bagul A, Nicholson ML. Hypothermic machine perfusion after static cold storage does not improve the preservation condition in an experimental porcine kidney model. Br J Surg. 2011;98(7):943-950.

38. Fuller BJ, Lee CY. Hypothermic perfusion preservation: the future of organ preservation revisited? Cryobiology. 2007;54(2):129-145. 


\section{Figure legends}

Figure 1: Kaplan-Meier analysis with up to 3 year follow-up data for different cold ischemia times. Multivariable $\mathrm{p}$ values derived from Cox models are displayed in each graph. 
Figure 1
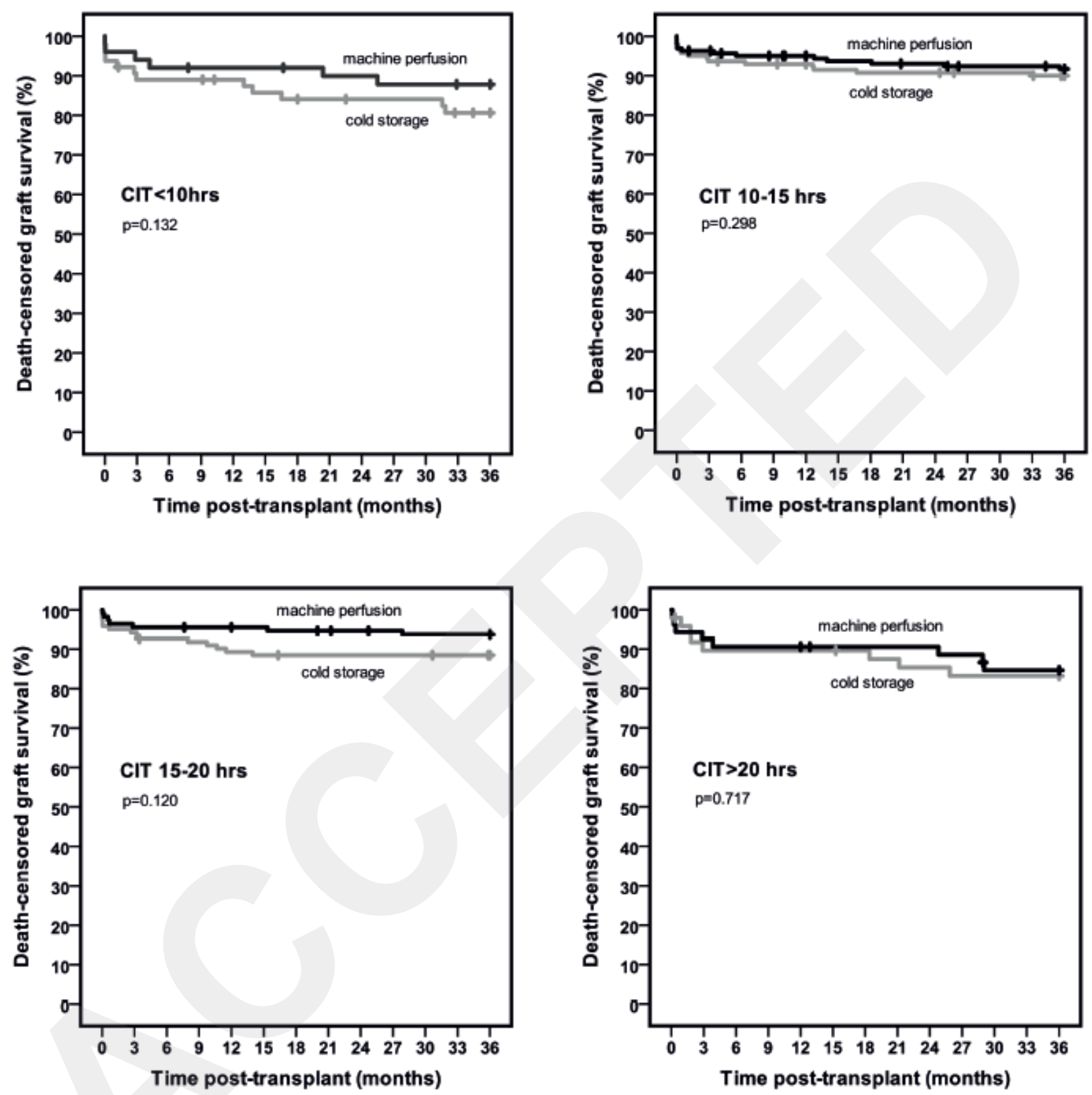
Table 1: Overall baseline study characteristics. Data are presented as median (range) or as number of patients.

HMP $\quad$ CS

\begin{tabular}{|c|c|c|c|}
\hline \multicolumn{4}{|l|}{ Donor characteristics } \\
\hline Age (yrs) & Median (range) & $49.6(16-81)$ & $49.6(16-81)$ \\
\hline \multicolumn{4}{|l|}{ Donor type } \\
\hline DBD & & 294 & 294 \\
\hline DCD category III & & 82 & 82 \\
\hline Standard criteria & & 271 & 271 \\
\hline Expanded criteria & & 105 & 105 \\
\hline \multicolumn{4}{|l|}{ Recipient characteristics } \\
\hline Age (yrs) & Median (range) & $53(11-79)$ & $52(2-79)$ \\
\hline Cold ischemia time (hrs:min) & & $15: 10(3-47)$ & $15: 05(3-30)$ \\
\hline
\end{tabular}


Table 2: Baseline characteristics split by cold ischemia time. Data are presented as number of cases (percentage) or median (range).

\begin{tabular}{|c|c|c|c|c|c|c|c|c|c|}
\hline \multirow[t]{2}{*}{$\mathrm{CIT}$ group } & & \multicolumn{2}{|c|}{$0-10$ hours } & \multicolumn{2}{|c|}{ 10-15 hours } & \multicolumn{2}{|c|}{$15-20$ hours } & \multicolumn{2}{|c|}{$20+$ hours } \\
\hline & & HMP & CS & HMP & CS & HMP & CS & HMP & CS \\
\hline Cohort size (no.) & & 50 & 64 & 160 & 141 & 113 & 122 & 53 & 49 \\
\hline Donor age (yrs) & Median (range) & $61(18-81)$ & $57(20-77)$ & $48.5(16-78)$ & $49(17-81)$ & $48(17-68)$ & $49(17-78)$ & $50(21-77)$ & $48(16-71)$ \\
\hline \multirow[t]{2}{*}{ Donor sex } & Male & 31 (62.0\%) & $37(47.8 \%)$ & 85 (53.1\%) & 80 (56.7\%) & 74 (65.5\%) & 72 (59.0\%) & 30 (56.6\%) & 31 (63.3\%) \\
\hline & Female & $19(38.0 \%$ & $27(42.2 \%)$ & 75 (46.9\%) & $61(43.3 \%)$ & 39 (34.5\%) & 50 (41.0\%) & 23 (43.4\%) & 18 (36.7\%) \\
\hline Recipient age (yrs) & Median (range) & $64(26-75)$ & $62,5(26-75)$ & $52(13-72)$ & $52(7-79)$ & 54 (11-79) & $49.5(2-77)$ & 49 (24-76) & $50(13-68)$ \\
\hline CIT (hrs:min) & Median (range) & $7: 31(4-10)$ & $8: 02(3-10)$ & $14: 10(10-15)$ & $13: 30(10-15)$ & $16: 59(15-20)$ & $16: 58(15-20)$ & $22: 39(20-30)$ & $22: 33(20-47)$ \\
\hline \multirow[t]{3}{*}{ Renal arteries (no.) } & 1 & $27(54.0 \%)$ & 38 (59.4\%) & 95 (59.4\%) & 70 (49.6\%) & $48(42.5 \%)$ & 58 (47.5\%) & 21 (39.6\%) & 26 (52.1\%) \\
\hline & 2 & $21(42.0 \%)$ & $22(34.4 \%)$ & 52 (32.5\%) & 64 (45.4\%) & $48(42.5 \%)$ & 47 (38.5\%) & $24(45.3 \%)$ & $11(22.4 \%)$ \\
\hline & ? & $2(4.0 \%)$ & $4(6.3 \%)$ & $13(8.1 \%)$ & $7(5.0 \%)$ & $17(15.0 \%)$ & 17 (13.9\%) & $8(15.1 \%)$ & $12(24.5 \%)$ \\
\hline \multirow[t]{2}{*}{ Donor type } & DBD & 47 (94.0\%) & 60 (93.8\%) & 131 (81.9\%) & $118(83.7 \%)$ & $80(70.8 \%)$ & 85 (69.7\%) & 36 (67.9\%) & $31(63.3 \%)$ \\
\hline & DCD & $3(6.0 \%)$ & $4(6.3 \%)$ & 29 (18.1\%) & $23(16.3 \%)$ & $33(29.2 \%)$ & 37 (30.3\%) & 17 (32.1\%) & $18(36.7 \%)$ \\
\hline \multirow[t]{2}{*}{ ECD donor } & Yes & $27(54.0 \%)$ & 31 (48.4\%) & 44 (27.5\%) & 34 (24.1\%) & $24(21.2 \%)$ & 29 (23.8\%) & 10 (18.9\%) & $11(77.6 \%)$ \\
\hline & No & $23(46.0 \%)$ & 33 (51.6\%) & $116(72.5 \%)$ & 107 (75.9\%) & 89 (78.8\%) & 93 (76.2\%) & 43 (81.1\%) & 38 (22.4\%) \\
\hline \multirow[t]{7}{*}{ HLA mismatches (no.) } & 0 & $5(10.0 \%)$ & $2(3.1 \%)$ & $23(14.4 \%)$ & $30(21.3 \%)$ & $18(15.9 \%)$ & 17 (13.9\%) & $9(17.0 \%)$ & $5(10.2 \%)$ \\
\hline & 1 & $1(2.0 \%)$ & $4(6.3 \%)$ & $14(8.8 \%)$ & $11(7.8 \%)$ & $6(5.3 \%)$ & $8(6.6 \%)$ & $10(18.9 \%)$ & $5(10.2 \%)$ \\
\hline & 2 & $13(26.0 \%)$ & $17(26.6 \%)$ & $43(16.9 \%)$ & $31(22.0 \%)$ & $31(27.4 \%)$ & $41(33.6 \%)$ & $11(20.8 \%)$ & $13(26.5 \%)$ \\
\hline & 3 & $13(26.0 \%)$ & $18(28.1 \%)$ & $48(30.0 \%)$ & 45 (31.9\%) & $42(37.2 \%)$ & 40 (32.8\%) & $16(30.0 \%)$ & $21(42.9 \%)$ \\
\hline & 4 & $9(18.0 \%)$ & $15(23.4 \%)$ & $24(15.0 \%)$ & $19(13.5 \%)$ & $14(12.4 \%)$ & $12(9.8 \%)$ & $4(7.5 \%)$ & $5(10.2 \%)$ \\
\hline & 5 & $4(8.0 \%)$ & $4(6.3 \%)$ & $3(1.9 \%)$ & $2(1.4 \%)$ & $2(1.8 \%)$ & $2(1.6 \%)$ & $2(3.8 \%)$ & $0(0.0 \%)$ \\
\hline & 6 & $5(10.0 \%)$ & $4(6.3 \%)$ & $5(3.1 \%)$ & $3(2.1 \%)$ & $0(0.0 \%)$ & $2(1.6 \%)$ & $1(1.9 \%)$ & $0(0.0 \%)$ \\
\hline Time spent on waiting list (yrs) & Median (range) & $5,5(1-8)$ & $6(1-8)$ & $5(1-8)$ & $5(1-8)$ & $5(1-8)$ & $4(1-8)$ & $5(1-8)$ & $5(2-8)$ \\
\hline \multirow[t]{3}{*}{ Previous transplants (no.) } & 0 & $40(80.0 \%)$ & $51(79.7 \%)$ & $110(68.8 \%)$ & $112(79.4 \%)$ & $76(67.3 \%)$ & 78 (63.9\%) & $34(64.2 \%)$ & $26(53.1 \%)$ \\
\hline & 1 & $10(20.0 \%)$ & $10(15.6 \%)$ & $42(26.3 \%)$ & $24(17.0 \%)$ & $33(29.2 \%)$ & $41(33.6 \%)$ & $13(24.5 \%)$ & $19(38.8 \%)$ \\
\hline & 2 & $0(0.0 \%)$ & $3(4.7 \%)$ & $7(4.4 \%)$ & $5(3.5 \%)$ & $3(2.7 \%)$ & $3(2.5 \%)$ & $4(7.5 \%)$ & $4(8.2 \%)$ \\
\hline
\end{tabular}




\begin{tabular}{|c|c|c|c|c|c|c|c|c|c|}
\hline & 3 & $0(0.0 \%)$ & $0(0.0 \%)$ & $1(0.6 \%)$ & 0 (0.0\%) & $1(0.9 \%)$ & $0(0.0 \%)$ & $1(1.9 \%)$ & $0(0.0 \%)$ \\
\hline & 4 & $0(0.0 \%)$ & $0(0.0 \%)$ & $0(0.0 \%)$ & $0(0.0 \%)$ & $0(0.0 \%)$ & $0(0.0 \%)$ & $1(1.9 \%)$ & $0(0.0 \%)$ \\
\hline \multirow[t]{5}{*}{ PRA } & $<5 \%$ & 48 (96.0\%) & 60 (93.8\%) & 137 (85.6\%) & 127 (90.0\%) & 112 (91.8\%) & 112 (91.8\%) & 47 (88.7\%) & 42 (85.7\%) \\
\hline & $5-25 \%$ & $2(4 \%)$ & $3(4.8 \%)$ & $14(8.8 \%)$ & $9(6.3 \%)$ & $5(4.1 \%)$ & 5 (4.1\%) & $0(0.0 \%)$ & $3(6.1 \%)$ \\
\hline & $25-50 \%$ & $0(0 \%)$ & $1(1.6 \%)$ & $6(3.8 \%)$ & $3(2.1 \%)$ & $2(1.6 \%)$ & 2 (1.6\%) & $2(3.8 \%)$ & $1(2.0 \%)$ \\
\hline & $50-85 \%$ & $0(0 \%)$ & $0(0 \%)$ & $3(1.8 \%)$ & $2(1.4 \%)$ & $1(0.8 \%)$ & $1(0.8 \%)$ & $2(3.8 \%)$ & $3(6.1 \%)$ \\
\hline & $>85 \%$ & $0(0 \%)$ & $0(0 \%)$ & $0(0.0 \%)$ & $0(0.0 \%)$ & $2(1.6 \%)$ & $2(1.6 \%)$ & $2(3.8 \%)$ & $0(0.0 \%)$ \\
\hline
\end{tabular}


Table 3: The incidence of DGF in the subgroup analysis. Data presented as number of cases and percentage of group total. Full multivariable models are provided in Table S1 ( $<10 \mathrm{hrs})$, Table S2 (10-15 hrs), Table S3 (15-20 hrs) and Table S4 (>20 hrs) (SDC, http://links.lww.com/TP/B552).

\begin{tabular}{l|ll|ll|l|l} 
CIT & Delayed graft function \\
\hline & HMP & CS & & Univariable & Multivariable OR (95\% Cl) \\
& $\mathrm{N}$ & $\%$ & $\mathrm{~N}$ & $\%$ & & \\
$\mathbf{< 1 0} \mathbf{~ h r s}$ & 3 & 6.0 & 18 & 28.1 & $\mathrm{p}=0.002$ & $0.015(0.001-0.317, \mathrm{p}=0.007)$ \\
$\mathbf{1 0 - 1 5} \mathbf{~ h r s}$ & 39 & 24.4 & 32 & 22.7 & $\mathrm{p}=0.593$ & $0.893(0.485-1.647, \mathrm{p}=0.718)$ \\
$\mathbf{1 5 - 2 0} \mathbf{~ h r s}$ & 31 & 27.4 & 42 & 34.4 & $\mathrm{p}=0.689$ & $0.663(0.355-1.240, \mathrm{p}=0.199)$ \\
$\mathbf{> 2 0} \mathbf{h r s}$ & 19 & 35.8 & 26 & 53.1 & $\mathrm{p}=0.08$ & $0.335(0.110-1.015, \mathrm{p}=0.053)$ \\
\hline Total & $\mathbf{9 2}$ & $\mathbf{2 4 . 5}$ & $\mathbf{1 1 8}$ & $\mathbf{3 1 . 4}$ & & \\
\hline
\end{tabular}


Variable

Hazard Ratio P value

(95\% Confidence interval)

\begin{tabular}{lll}
\hline Donor age (yrs) & $1.093(0.997-1.199)$ & 0.057 \\
Recipient age (yrs) & $0.991(0.926-1.062)$ & 0.804 \\
\hline Machine perfusion vs cold storage & $0.460(0.168-1.264)$ & 0.132 \\
\hline Cold ischemia time (hrs) & $1.036(0.761-1.412)$ & 0.821 \\
\hline DCD donor vs DBD donor & 0.000 & 0.984 \\
Expanded criteria vs standard criteria & $0.122(0.008-1.812)$ & 0.126 \\
\hline HLA mismatches (no.) & $1.905(1.162-3.121)$ & 0.011 \\
\hline Panel-reactive antibody level (\%) & $0.945(0.763-1.171)$ & 0.607 \\
\hline Duration of pretransplant dialysis (yrs) & $0.875(0.669-1.144)$ & 0.329 \\
\hline Retransplantation vs first transplantation & $2.086(0.730-5.963)$ & 0.170 \\
\hline
\end{tabular}

Table S1: Multivariate Cox risk analysis for delayed graft function. Cold ischemia time $<10$ hours.

\begin{tabular}{lll} 
Variable & $\begin{array}{l}\text { Hazard Ratio } \\
\text { (95\% Confidence interval) }\end{array}$ & P value \\
\hline Donor age (yrs) & $1.022(0.972-1.075)$ & 0.387 \\
\hline Recipient age (yrs) & $0.998(0.967-1.030)$ & 0.916 \\
\hline Machine perfusion vs cold storage & $0.659(0.301-1.446)$ & 0.298 \\
\hline Cold ischemia time (hrs) & $1.187(0.886-1.590)$ & 0.252 \\
\hline DCD donor vs DBD donor & $1.274(0.395-4.111)$ & 0.686 \\
\hline Expanded criteria vs standard criteria & $2.758(0.818-9.297)$ & 0.102 \\
\hline HLA mismatches (no.) & $1.023(0.785-1.334)$ & 0.866 \\
\hline Panel-reactive antibody level (\%) & $0.987(0.944-1.033)$ & 0.585 \\
\hline Duration of pretransplant dialysis (yrs) & $0.962(0.806-1.148)$ & 0.666 \\
\hline Retransplantation vs first transplantation & $1.635(0.927-2.883)$ & 0.089 \\
\hline
\end{tabular}

Table S2: Multivariate Cox risk analysis for delayed graft function. Cold ischemia time between 10 and 15 hours. 


\begin{tabular}{lll} 
Variable & $\begin{array}{l}\text { Hazard Ratio } \\
\text { (95\% Confidence interval) }\end{array}$ & P value \\
\hline Donor age (yrs) & $1.085(1.015-1.160)$ & 0.0168 \\
Recipient age (yrs) & $0.981(0.948-1.016)$ & 0.2791 \\
\hline Machine perfusion vs cold storage & $0.469(0.180-1.220)$ & 0.1204 \\
\hline Cold ischemia time (hrs) & $1.018(0.754-1.376)$ & 0.9052 \\
\hline DCD donor vs DBD donor & $0.922(0.315-2.701)$ & 0.8826 \\
\hline Expanded criteria vs standard criteria & $0.354(0.085-1.476)$ & 0.1540 \\
\hline HLA mismatches (no.) & $0.942(0.667-1.331)$ & 0.7349 \\
\hline Panel-reactive antibody level (\%) & $1.016(0.996-1.035)$ & 0.1142 \\
\hline Duration of pretransplant dialysis (yrs) & $1.109(0.890-1.381)$ & 0.3564 \\
\hline Retransplantation vs first transplantation & $1.423(0.724-2.798)$ & 0.3065 \\
\hline
\end{tabular}

Table S3: Multivariate Cox risk analysis for delayed graft function. Cold ischemia time between 15 and 20 hours.

\begin{tabular}{lll} 
Variable & $\begin{array}{l}\text { Hazard Ratio } \\
\text { (95\% Confidence interval) }\end{array}$ & P value \\
\hline Donor age (yrs) & $0.996(0.941-1.055)$ & 0.900 \\
Recipient age (yrs) & $0.958(0.915-1.004)$ & 0.074 \\
\hline Machine perfusion vs cold storage & $0.824(0.290-2.342)$ & 0.717 \\
\hline Cold ischemia time (hrs) & $0.922(0.782-1.087)$ & 0.333 \\
\hline DCD donor vs DBD donor & $1.290(0.369-4.506)$ & 0.690 \\
\hline Expanded criteria vs standard criteria & $3.471(0.744-16.198)$ & 0.113 \\
\hline HLA mismatches (no.) & $1.484(0.957-2.300)$ & 0.078 \\
\hline Panel-reactive antibody level (\%) & $1.030(1.000-1.061)$ & 0.050 \\
\hline Duration of pretransplant dialysis (yrs) & $0.985(0.753-1.288)$ & 0.911 \\
\hline Retransplantation vs first transplantation & $0.588(0.203-1.700)$ & 0.327 \\
\hline
\end{tabular}

Table S4: Multivariate Cox risk analysis for delayed graft function. Cold ischemia time longer than 20 hours. 